\title{
Perfil de usuários da atenção primária acerca do serviço de eletrocardiograma com laudo por telecardiologia
}

\begin{abstract}
RESUMO | Objetivo: identificar o perfil dos usuários da Atenção Primária acerca do serviço de eletrocardiograma com laudo por telecardiologia. Método: trata-se de um artigo oriundo de um trabalho de conclusão de curso (monografia), estudo descritivo, exploratório, documental, com abordagem quantitativa, realizado com 31 pacientes de um município de Minas Gerais que realizaram eletrocardiograma com laudo por telecardiologia em uma Estratégia de Saúde da Família. A busca dos dados foi realizada durante $01^{\circ}$ semestre de 2018, entre os meses de março e maio. Os dados foram discutidos conforme epidemiologia descritiva simples uni-variada não paramétrica e não probabilística. Resultados: houve prevalência do sexo feminino, idade média de 49,8 anos e sobrepeso. As comorbidades autorreferidas mais prevalentes foram a hipertensão e o histórico familiar. A dor torácica, queimação, pontadas, formigamento e pressão arterial descompensada foram as principais queixas para a realização do exame e o diagnóstico mais prevalente foi a normalidade. Conclusão: apesar da maior parte dos exames não terem evidenciado alterações, as variáveis socioeconômicas, metabólicas e genéticas constituem fator de risco para o desenvolvimento de doenças cardiovasculares.

Descritores: Eletrocardiograma; Telemedicina; Cardiopatias; Atenção primária a saúde.
\end{abstract}

\begin{abstract}
Objective: to identify the profile of primary care users about the electrocardiogram service with telecardiology reports. Method: this is an article originated from a course conclusion work (monograph), this is a descriptive, exploratory, documentary study with a quantitative approach, conducted with 31 patients from a city from Minas Gerais who underwent electrocardiogram with telecardiology report in a Family Health Strategy. The data search was conducted during the 1st semester of 2018, between the months of March and May. The data were discussed according to simple descriptive epidemiology univariate non-parametric and non-probabilistic. Results: There was a prevalence of female gender, mean age of 49.8 years and overweight. The most prevalent self-reported comorbidities were hypertension and family history. Chest pain, burning, pricking, tingling and decompensated blood pressure were the main complaints for the exam and the most prevalent diagnosis was normality. Conclusion: despite the fact that most of the exams showed no alterations, socioeconomic, metabolic and genetic variables are risk factors for the development of cardiovascular diseases.
\end{abstract}

Descriptors: Electrocardiography; Telemedicine; Heart diseases; Primary health care.

RESUMEN | Objetivo: identificar el perfil de usuarios de atención primaria sobre el servicio de electrocardiograma con informe de telecardiología. Método: este es un artículo resultante de un trabajo de finalización de curso (monografía), estudio descriptivo, exploratorio, documental, con enfoque cuantitativo, realizado con 31 pacientes de una ciudad de Minas Gerais a los que se les realizó electrocardiograma con informe de telecardiología en una Estrategia de Salud de la Familia. La búsqueda de datos se realizó durante el 1er semestre de 2018, entre los meses de marzo y mayo. Los datos se analizaron según la epidemiología descriptiva simple uni-variante no paramétrica y no probabilística. Resultados: hubo prevalencia de sexo femenino, edad media de 49,8 años y sobrepeso. Las comorbilidades autorreferidas más prevalentes fueron la hipertensión y la historia familiar. El dolor torácico, ardor, pinchazo, hormigueo y descompensación de la presión arterial fueron las principales quejas para realizar el examen y el diagnóstico más prevalente fue la normalidad. Conclusión: aunque la mayoría de los exámenes no mostraron cambios, las variables socioeconómicas, metabólicas y genéticas constituyen un factor de riesgo para el desarrollo de enfermedades cardiovasculares.

Palabras claves: Electrocardiografía; Telemedicina; Cardiopatías; Atención primaria de salud.

\section{Patrick Leonardo Nogueira da Silva}

Enfermeiro, Mestrando pelo Programa de Pós-Graduação em Cuidado Primário em Saúde da Universidade Estadual de Montes Claros (PPGCPS/UNIMONTES). Montes Claros, MG, Brasil.

ORCID: 0000-0003-2399-9526

\section{Bárbara Nogueira Silva}

Enfermeira pela Faculdade de Saúde e Desenvolvimento Humano Santo Agostinho (FASA). Montes Claros, MG, Brasil. ORCID: 0000-0001-6765-9684

\section{Dennerson Rodrigues Emerich da Silva}

Enfermeiro pela Faculdade de Saúde e Desenvolvimento Humano Santo Agostinho (FASA). Montes Claros, MG, Brasil.

ORCID: 0000-0001-5669-138X

\section{Cláudio Luís de Souza Santos}

Enfermeiro, Especialista em Saúde Mental pela Residência Multiprofissional da Universidade Estadual de Montes Claros (UNIMONTES). Montes Claros, MG, Brasil.

ORCID: 0000-0002-9127-6349

Recebido em: 09/12/2021

Aprovado em: 15/01/2022

\section{Thiago Santos Monção}

Enfermeiro, Mestre em Gestão de Tecnologia e Inovação em Saúde, Servidor Público da Prefeitura de Montes Claros (PMMC). Montes Claros, MG, Brasil. ORCID: 0000-0002-1683-4478

\section{Carolina dos Reis Alves}

Enfermeira, Doutora em Ciências da Saúde, Professora do Departamento de Enfermagem da Faculdade de Saúde e Desenvolvimento Humano Santo Agostinho (FASA). Montes Claros, MG, Brasil.

ORCID: 0000-0003-2107-6306 


\author{
INTRODUÇÃO
}

$\wedge$. Atenção Primária à Saúde (APS), no âmbito legislativo do Sistema Único de Saúde (SUS), está organizada como porta de entrada preferencial ao sistema. Na concepção desse nível assistencial, o cuidado centrado no usuário deve responder a todas as necessidades de saúde da população por meio de um sistema integrado, articulado e pautado na construção de redes de trabaIho intersetoriais(1).

A Unidade Básica de Saúde (UBS) tem seu campo de trabalho considerado de baixa densidade e de alta complexidade, lidando diariamente com uma grande variedade de problemas, desde aqueles gerados pelas mazelas sociais que requerem um trabalho intersetorial para a construção de respostas, até aquelas situações clínicas que demandam ações nas quais a própria equipe de saúde deve estar articulada para responder de forma resolutiva(2).

A telecardiologia, em especial a realização de eletrocardiograma (ECG), permite uma atenção maior às doenças cardiovasculares (DCV), principal causa de óbito no Brasil. Tal exame torna-se um importante método para averiguação, constatação e condução de DCV(3).

O Projeto Minas Telecardio, iniciado em 2006 no Estado de Minas Gerais, disponibilizou equipamento para a realização de ECG digital para 82 municípios de pequeno porte, sendo que os exames de ECG digital são laudados por médicos cardiologistas nos polos de telecardiologia nas universidades participantes do projeto e, dessa maneira, proporcionando a realização do exame laudado por especialista a um custo inferior aos métodos tradicionais(4).

A integração entre o médico de família e o cardiologista no acompanhamento das DCV permite maior qualidade no atendimento prestado auxiliando na detecção de critérios para

\section{A Unidade Básica de Saúde (UBS)} tem seu campo de trabalho considerado de baixa densidade e de alta complexidade, lidando diariamente com uma grande variedade de problemas, desde aqueles gerados pelas mazelas sociais

que requerem um trabalho intersetorial para a construção de respostas, até aquelas situações clínicas que demandam ações nas quais a própria equipe de saúde deve estar articulada para responder de forma resolutiva encaminhamento do paciente à consulta especializada, seguindo as diretrizes terapêuticas. Além disso, permite maior estreitamento entre os profissionais com a identificação dos casos graves e discussão dos mesmos, redução dos encaminhamentos, solicitação de exames complementares e finalmente funcionando como uma ferramenta de ensino em serviço(5).

Os eventos cardiovasculares podem ser predispostos por fatores extrínsecos cujos quais podem ser modificados, e fatores intrínsecos ou não modificáveis. Os hábitos de vida refletem em longo prazo no surgimento desses eventos, bem como no agravamento dos fatores de risco de modo a aumentar a prevalência de comorbidades(6-9). Diante disso, o problema posto para investigação tem a seguinte questão norteadora: Qual o perfil dos usuários da APS acerca do serviço de ECG com laudo por telecardiologia?

Considerando o impacto das DCV e possíveis dificuldades para o acesso ao exame de ECG na APS, o presente estudo objetivou analisar o perfil dos usuários da APS acerca do serviço de ECG com laudo por telecardiologia.

\section{MÉTODOS}

Artigo da monografia intitulada "Percepção dos usuários da Atenção Primária do Município de Montes Claros - MG acerca do serviço de eletrocardiograma (ECG) com laudo por telecardiologia", apresentada ao Departamento de Enfermagem da Faculdade Santo Agostinho/FASA. Montes Claros (MG), Brasil. 2018.

Trata-se de um estudo descritivo, exploratório, documental, com abordagem quantitativa, realizado na Estratégia de Saúde da Família (ESF) do Bairro Vera Cruz, localizado no município de Montes Claros, Minas Gerais, cujo qual apresentava o serviço de ECG disponível à população. Além deste bairro, outros pólos ofertavam deste serviço 
cujos quais se localizavam nos seguintes bairros do referido município: Major Prates, Alto São João e Maracanã. A amostra foi composta por 31 pacientes, sendo estes usuários da APS de Montes Claros, os quais realizaram o ECG com laudo na ESF. Ainda, foram utilizadas as informações contidas nos laudos dos respectivos pacientes para compor os dados do estudo.

Foi enviada uma carta de apresentação e um Termo de Consentimento Institucional $(\mathrm{TCl})$ à Coordenação da Atenção Básica (AB) da Secretaria Municipal de Saúde de Montes Claros (SMS/MOC) para autorização do estudo. A instituição foi devidamente orientada quanto às diretrizes da pesquisa, com assinatura de ciência autorizando a pesquisa. A busca dos dados foi realizada durante o $1^{\circ}$ semestre de 2018 pelo pesquisador responsável, entre os meses de março e maio, em uma Unidade Básica de Saúde onde havia a realização do ECG digital com laudo.

Foram adotados os seguintes critérios de inclusão para participação no estudo: (1) ter mais de 18 anos; (2) residir no município de Montes Claros; (3) ter a prescrição médica com a justificativa especificada para a realização do ECG. O estudo foi concentrado apenas na ESF Vera Cruz tendo em vista que a mesma era a ESF de referência deste serviço para todo o município, enquanto que os demais pólos que também dispunham do serviço foram excluídos da pesquisa em decorrência dos mesmos atenderem apenas a sua própria população adscrita. Para a realização do estudo, foi elaborado um roteiro de questionário semiestruturado o qual foi utilizado durante a abordagem dos usuários quanto à realização do exame associado ao seu resultado. As variáveis independentes da pesquisa foram constituídas pelo sexo, média da idade, dados antropométricos (peso, altura e IMC), comorbidades, motivo (queixa, justificativa) e conclusão do resultado.
Após a coleta, os dados foram armazenados e organizados em um banco de dados utilizando-se do programa estatístico Statistical Package for the Social Science (SPSS), versão 18.0®. Os achados do estudo foram apresentados por meio de tabelas elaboradas no Microsoft Excel@, Windows for Windows, versão 2013, contendo freqüências absolutas e percentuais e, em seguida, foram submetidos à análise descritiva.

Os participantes foram devidamente orientados quanto às diretrizes do estudo na qual os mesmos assinaram o Termo de Consentimento Livre e Esclarecido (TCLE) de modo a autorizar a realização da pesquisa. Os participantes tiveram resguardadas as suas identidades pessoais, de modo a ser garantido o sigilo e o anonimato no estudo.

O estudo obedeceu aos preceitos éticos estabelecidos pela Resolução $\mathrm{n}^{\circ}$ 466, de 12 de dezembro de 2012, do Conselho Nacional de Saúde (CNS), o qual regulamenta a realização da pesquisa envolvendo seres humanos. O projeto de pesquisa foi apreciado e aprovado pelo Comitê de Ética em Pesquisa da Universidade Estadual de Montes Claros (CEP UNIMONTES), sob o parecer consubstanciado $\mathrm{n}^{\circ}$ 2.029.185/2017, Certificado de Apresentação para Apreciação Ética (CAAE) $n^{\circ}$ 64404716.2.0000.5146.

\section{RESULTADOS}

Dos exames analisados, observou-se prevalência de pacientes do sexo feminino $(67,7 \%)$, idade média geral de 49,8 anos e pacientes com sobrepeso $(58,1 \%)$ (Tabela 1$)$. Constata-se que, embora haja prevalência de pacientes do sexo feminino na amostra do estudo, o sexo masculino apresenta os maiores índices massa corporal (IMC) quanto às classificações de sobrepeso e obesidade grau II.

Com base nos dados avaliados no exame, também foi possível traçar um perfil epidemiológico destes pacientes 
por meio das comorbidades identificadas no ECG. Observa-se um quantitativo significativo da hipertensão arterial sistêmica (HAS) $(54,8 \%)$ e da influência do histórico familiar de doença coronariana $(38,8 \%)$ sobre o sexo feminino (Tabela 2).

Quanto aos critérios de indicação para realização do exame, a presença de sintomas relacionados à dor torácica, queimação, pontadas, formigamento precordial e HAS descompensada (25,7\%). É importante salientar o tempo médio de espera (TME) entre a solicitação do exame e a realização, sendo que, nesses sintomas mais urgentes, apresenta-se em torno de 73,3 dias para homens e 20,4 dias para mulheres (Tabela 3).

Ao realizar a análise dos exames alterados buscando sua correlação com critérios de idade, IMC, comorbidades relacionadas e presença de histórico familiar de doença coronariana, foi possível observar nitidamente que na população feminina a idade estava entre 39 e 80 anos. Além disso, 95\% das alterações tinham correlação com sobrepeso, HAS e, além disso, 50\% delas possuíam histórico familiar de doença coronariana.

Nos homens, a idade estava entre os 32 e 74 anos, a expressão do IMC estava presente de forma reservada com apenas $30,0 \%$ de representatividade no sobrepeso, entretanto quando associado ao histórico familiar, 90,0\% dessa população era predisposta para esse fator de risco.

Dentre os resultados dos exames analisados, 58, $1 \%$ encontravam-se alterados. Destes, 55,0\% tinham apenas uma alteração, seguidos por 27,7\% com duas alterações e $16,6 \%$ com três alterações. Esses dados representam um valor de 18 conclusões eletrocardiográficas que resultaram em 14 diagnósticos distintos que foram estratificados na Tabela 4. A principal alteração eletrocardiográfica foi de repolarização ventricular inespecífica, que cor-
Tabela 1 - Perfil descritivo da população atendida para realização de ECG com laudo por telecardiologia na APS. Montes Claros, MG, Brasil. ( $n=31)$

\begin{tabular}{|c|c|c|c|c|c|c|c|c|c|}
\hline \multirow{3}{*}{ Variável } & \multicolumn{9}{|c|}{ Frequência } \\
\hline & \multicolumn{3}{|c|}{ Masculino } & \multicolumn{3}{|c|}{ Feminino } & \multicolumn{3}{|c|}{ Geral } \\
\hline & $\mathrm{n}$ & $\%$ & MA & $\mathrm{n}$ & $\%$ & MA & $\mathrm{n}$ & $\%$ & MA \\
\hline Sexo & 10 & 32,3 & - & 21 & 67,7 & - & 31 & 100 & - \\
\hline Idade (anos) & - & - & 53,0 & - & - & 50,2 & - & - & 49,8 \\
\hline Peso (kg) & - & - & 43,5 & - & - & 81,5 & - & - & 68,5 \\
\hline Altura $(\mathrm{cm})$ & - & - & 168 & - & - & 155 & - & - & 159 \\
\hline IMC (kg/m2) & - & - & 31,8 & - & - & 24,7 & - & - & 29,8 \\
\hline Baixo peso & 00 & 0,0 & - & 02 & 6,5 & - & 02 & 6,5 & - \\
\hline Normal & 03 & 9,6 & - & 04 & 12,9 & - & 07 & 22,5 & - \\
\hline Sobrepeso & 05 & 16,2 & - & 13 & 41,9 & - & 18 & 58,1 & - \\
\hline Obesidade grau I & 00 & 0,0 & - & 01 & 3,2 & - & 01 & 3,2 & - \\
\hline Obesidade grau II & 02 & 6,5 & - & 00 & 0,0 & - & 02 & 6,5 & - \\
\hline Obesidade grau III & 00 & 0,0 & - & 01 & 3,2 & - & 01 & 3,2 & - \\
\hline
\end{tabular}

Fonte: Dados da pesquisa, 2018. MA = Média Aritmética.

Tabela 2 - Comorbidades autorreferidas pela população atendida para realização de ECG com laudo por telecardiologia no serviço de APS. Montes Claros, MG, Brasil. $(n=31)$

\begin{tabular}{cccccccc} 
Comorbidades autorreferidas & \multicolumn{3}{c}{ Masculino } & \multicolumn{2}{c}{ Feminino } & \multicolumn{2}{c}{ Geral } \\
& $n$ & $\%$ & $n$ & $\%$ & $n$ & $\%$ \\
HAS & 06 & 19,4 & 11 & 35,4 & 17 & 54,8 \\
IAM prévio & 01 & 3,2 & 00 & 0,0 & 01 & 3,2 \\
DRC & 00 & 0,0 & 00 & 0,0 & 00 & 0,0 \\
DC & 00 & 0,0 & 00 & 0,0 & 00 & 0,0 \\
CRVM prévia & 00 & 0,0 & 00 & 0,0 & 00 & 0,0 \\
Tabagismo & 00 & 0,0 & 00 & 0,0 & 00 & 0,0 \\
DM & 00 & 0,0 & 01 & 3,2 & 01 & 3,2 \\
Doença Pulmonar Crônica & 00 & 0,0 & 00 & 0,0 & 00 & 0,0 \\
\hline Histórico familiar de doença coronariana & 03 & 9,7 & 09 & 29,1 & 12 & 38,8
\end{tabular}

Fonte: Dados da pesquisa, 2018. HAS = Hipertensão Arterial Sistêmica. IAM = Infarto Agudo do Miocárdio. DRC $=$ Doença Renal Crônica. DC = Doença de Chagas. CRVM = Cirurgia de Revascularização do Miocárdio. DM = Diabetes Mellitus.

Tabela 3 - Estratificação de indicação para o ECG e tempo médio de espera para sua realização. Montes Claros, MG, Brasil. ( $n=31)$

\begin{tabular}{cccccccc} 
Motivo & \multicolumn{3}{c}{ Masculino } & \multicolumn{3}{c}{ Feminino } \\
& $\mathbf{n}$ & $\%$ & TME & N & $\%$ & TME \\
\hline $\begin{array}{c}\text { Dor torácica, queimação, pontadas, formiga- } \\
\text { mento e PA descompensada }\end{array}$ & 03 & 9,6 & 73,3 & 05 & 16,1 & 20,4 \\
$\begin{array}{c}\text { Dispnéia freqüente } \\
\text { HAS/PA descompensada }\end{array}$ & 00 & 0,0 & 0,0 & 02 & 6,7 & 105,0 \\
\hline
\end{tabular}


responde a $22,2 \%$ das conclusões.

Os achados desse estudo estão intrinsecamente correlacionados com todos os fatores de risco observados, sendo eles modificáveis (obesidade) e não modificáveis (sexo, idade, estatura), bem como com as comorbidades autorreferidas, de modo a propiciar e justificar o surgimento de toda a sintomatologia apresentada, tendo em vista o agravamento das manifestações clínicas (eventos cardiovasculares) diagnosticadas pelo ECG.

\section{DISCUSSÃO}

No presente estudo, observou-se prevalência do sexo feminino $(67,7 \%)$. Esse achado já era esperado tendo em vista as hipóteses do estudo, de modo a ser corroborado em outros dois estudos, um realizado em 2012 na cidade de Criciúma (Santa Catarina) com a análise de 1.949 laudos de ECG realizados em uma UBS, sendo que, destes, $1.230(63,1 \%)$ eram do sexo feminino, e outro no ano de 2013, com análise de 161 traçados de ECG com, aproximadamente, $57,8 \%$ de pacientes do sexo feminino $(6,7)$.

Segundo os últimos dados do Ministério da Saúde (MS), as DCV são responsáveis por grande parte da mortalidade dos brasileiros, correspondendo a $31 \%$ do total de óbitos, sendo proporcionalmente maior nas mulheres quando comparado aos homens(8). A idade média encontrada foi de 49,86 anos para ambos os sexos. Achados semelhantes foram encontrados em outros estudos os quais foram realizados em populações sem patologias prévias e com 34.371 pacientes que tinham a idade média entre 35 e 54 anos $(9,10)$.

A idade configura um fator de risco para o desenvolvimento da HAS, bem como para a aquisição de uma DCV. Geralmente são acometidas mulheres de 45 a 55 anos, período que marca o início do climatério (período de transição da fase reprodutiva para a fase de

\begin{tabular}{ccccccc} 
Risco cirúrgico & 00 & 0,0 & 0,0 & 05 & 16,1 & 25,0 \\
\hline Acompanhamento de rotina (arritmias, angina) & 03 & 9,6 & 18,3 & 04 & 12,5 & 127,5 \\
\hline IAM prévio & 01 & 3,2 & 60,0 & 00 & 0,0 & 0,0 \\
Não soube informar & 02 & 6,7 & 30,0 & 02 & 6,7 & 16,5 \\
Total & 10 & 32,3 & - & 21 & 67,7 & -
\end{tabular}

Fonte: Dados da pesquisa, 2018. HAS = Hipertensão Arterial Sistêmica. IAM = Infarto Agudo do Miocárdio. DRC $=$ Doença Renal Crônica. DC = Doença de Chagas. CRVM = Cirurgia de Revascularização do Miocárdio. DM = Diabetes Mellitus.

Tabela 4 - Descrição das conclusões dos resultados de ECG. Montes Claros, MG, Brasil. ( $n=31)$

\begin{tabular}{|c|c|c|c|c|c|c|}
\hline \multirow{2}{*}{ Conclusão } & \multicolumn{2}{|c|}{ Masculino } & \multicolumn{2}{|c|}{ Feminino } & \multicolumn{2}{|c|}{ Geral } \\
\hline & $n$ & $\%$ & $n$ & $\%$ & $n$ & $\%$ \\
\hline Intervalo PR curto & 00 & 0,0 & 02 & 6,4 & 02 & 6,4 \\
\hline Bloqueio intraventricular inespecífico & 00 & 0,0 & 01 & 3,2 & 01 & 3,2 \\
\hline Bloqueio divisional ântero-superior esquerdo & 01 & 3,2 & 02 & 6,4 & 03 & 9,6 \\
\hline Intervalo QT prolongado & 00 & 0,0 & 01 & 3,2 & 01 & 3,2 \\
\hline DLN para sexo e idade & 03 & 9,6 & 10 & 32,2 & 13 & 41,8 \\
\hline Distúrbio de condução do ramo direito & 02 & 6,4 & 01 & 3,2 & 03 & 9,6 \\
\hline $\begin{array}{c}\text { Alterações inespecíficas da repolarização } \\
\text { ventricular }\end{array}$ & 01 & 3,2 & 03 & 9,6 & 04 & 12,8 \\
\hline Taquicardia sinusal & 00 & 0,0 & 02 & 6,4 & 02 & 6,4 \\
\hline Sobrecarga atrial esquerda & 00 & 0,0 & 01 & 3,2 & 01 & 3,2 \\
\hline $\begin{array}{c}\text { Progressão lenta de onda R nas derivações } \\
\text { precordiais }\end{array}$ & 01 & 3,2 & 01 & 3,2 & 02 & 6,4 \\
\hline Bloqueio de ramo esquerdo & 01 & 3,2 & 02 & 6,4 & 03 & 9,6 \\
\hline $\begin{array}{c}\text { Área eletricamente inativa podendo estar } \\
\text { associada a IAM prévio }\end{array}$ & 02 & 6,4 & 02 & 6,4 & 04 & 12,8 \\
\hline Bloqueio atrioventricular de $1^{\circ} \mathrm{grau}$ & 01 & 3,2 & 00 & 0,0 & 01 & 3,2 \\
\hline Bloqueio do ramo direito & 01 & 3,2 & 00 & 0,0 & 01 & 3,2 \\
\hline
\end{tabular}

Fonte: Dados da pesquisa, 2018. DLN = Dentro dos Limites de Normalidade. IAM = Infarto Agudo do Miocárdio.

pós-menopausa), quando a produção de estrogênio pelos ovários decresce gradativamente. Desta forma, a mulher perde a importante proteção cardiovascular promovida pelo estrogênio. Diferentemente do sexo masculino cuja prevalência de DCV se dá antes dos 50 anos em decorrência de outros fatores de risco, tais como o tabagismo, etilismo, obesidade, maus hábitos alimentares, atividades laborais de maior esforço físico, dentre outros.

Quanto ao IMC, foi possível observar que $58,0 \%$ dos pacientes apre- sentavam sobrepeso. Infere-se quanto a esse achado que a população tem aumentado a predisposição para o sedentarismo e, conseqüentemente, para o desenvolvimento da obesidade em longo prazo. Ainda, existem dois grupos de fatores de risco, sendo eles os não modificáveis e os modificáveis por meio de intervenções no estilo de vida como é o caso do sobrepeso e obesidade(11). Ainda que o sedentarismo não tenha sido estratificado no panorama nacional, há algum tempo ele vem sendo mencionado por diversos autores 
como um importante fator de risco para as DCV(12).

Visando identificar o perfil epidemiológico dos usuários do estudo, investigou-se a presença de comorbidades pré-existentes e constatou-se a prevalência do histórico familiar para a doença coronariana em $67,7 \%$ dos participantes do estudo seguido por HAS em $54,8 \%$. Diante desse fato, é possível inferir que, ainda que o paciente não possua HAS, ele pode possuir um histórico familiar de modo a influenciar na predisposição para a doença. Fato esse que corrobora com um estudo epidemiológico cujo qual enfatiza os fatores genéticos, sendo esses responsáveis por $30 \%$ na variação da pressão arterial (PA) em populações distintas, e que a HAS seja duas vezes mais frequente em sujeitos com um de seus progenitores hipertensos(13).

Quanto aos critérios de indicação para realização do exame, observa-se presença de sintomas correlacionados à dor, queimação e formigamento em região precordial, HAS descompensada e dispnéia freqüente. A identificação desses fatores repercute diretamente na preocupação com o tempo de espera entre a solicitação do exame e a sua realização tendo em vista as complicações das queixas, sendo que, nesses sintomas mais urgentes o tempo médio de espera gira em torno de 20,4 dias para as mulheres e 73,3 dias para os homens. O longo tempo de espera pode acarretar vários problemas para os pacientes, seus familiares e a sociedade. Para os pacientes, pode ocorrer o agravamento da doença, podendo chegar ao óbito, podem surgir problemas psicológicos e repercussões para suas famílias(14).

No tocante às alterações eletrocardiográficas, o percentual de exames que se encontravam anormais variam geralmente entre 20 e $88,9 \%$, sendo que, no presente estudo, esse percentil era de 58,1\%. Embora tenham sido encontrados vários tipos de anormalida- des nos exames alterados, prevaleceu a Alteração da Repolarização Ventricular Inespecífica (ARVI). Sua avaliação por

As mulheres obesas
acima dos 50 anos apresentam maior predisposição às DCV quando comparadas aos homens, pois após esta idade, a mulher apresenta hipoestrogenismo, característica responsável pelo surgimento da menopausa, bem como ao aumento do risco para o desenvolvimento das doenças crônicas<smiles>[CH]1[CH]C=C1</smiles>

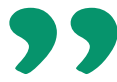

meio de ECG é extremamente complexa, pois esta representa a interação de vários sistemas capazes de se expres- sarem nos segmentos e ondas elétri$\operatorname{cas}(15)$.

O fenômeno da repolarização ganhou maior notoriedade ao trazer contribuições para a estratificação de risco de eventos arrítmicos graves e morte súbita. Nas últimas décadas, os grandes avanços foram as definições da dispersão da repolarização ventricular, marcador da recuperação não uniforme da excitabilidade miocárdica e o reconhecimento da macro ou da microalternância cíclica da onda $\mathrm{T}$, esse dado vai de encontro com achados de estudos que buscaram verificar os principais resultados de anormalidades em $\operatorname{ECG}(6,15)$.

Além disso, é importante salientar que este é um fator a se considerar uma vez que um estudo realizado na cidade de Cotia (São Paulo) no ano de 2002 concluiu que a ARVI era um fator de risco para doença isquêmica, principalmente em pacientes do sexo feminino(15).

\section{CONCLUSÃO}

O ineditismo temático configura uma limitação do estudo, tendo em vista que na literatura científica não apresenta outros estudos recentes abordando o tema. Ainda, a avaliação do paciente pós-diagnóstico limita o estudo quanto a uma abordagem mais detalhada. Foi possível notar que, devido a uma sobrecarga na agenda dos serviços, muitas vezes o tempo de espera para realização do exame é extenso, um fator preocupante, pois interfere diretamente no diagnóstico e cuidado.

As mulheres obesas acima dos 50 anos apresentam maior predisposição às DCV quando comparadas aos homens, pois após esta idade, a mulher apresenta hipoestrogenismo, característica responsável pelo surgimento da menopausa, bem como ao aumento do risco para o desenvolvimento das doenças crônicas, tal como a HAS, cardiopatias, infarto agudo do miocárdio 
(IAM), dentre outras. Sendo assim, com o processo de senescência, as mudanças no ritmo cardíaco se tornam perceptíveis por meio da realização do ECG.

Portanto, apesar da maior parte dos exames não terem evidenciado alterações, as variáveis socioeconômicas (sexo, idade), metabólicas (peso, IMC) e genéticas (histórico familiar) constituem fator de risco para o desenvolvimento de DCV. Sugere-se então, a realização de mais estudos que identifiquem e avaliem o perfil desse paciente antes, durante e após a realização do exame e do diagnóstico, visando à mudança dos fatores de risco modificáveis de modo a repercutir em um melhor prognóstico para o paciente.

\section{Referências}

1. Barbosa MAF, Marra VR, Horta NC, Rodrigues ES. Capacitação dos profissionais de saúde para o atendimento de parada cardiorrespiratória na atenção primária. Rev. APS [Internet]. 2011 [cited 2017 Nov 4];14(2):233-8. Available from: https://periodicos.ufjf.br/index.php/aps/ article/view/14699

2. Lumer S. 0 papel da saúde da família na atenção às urgências. Rev. APS [Internet]. 2011 [cited 2017 Nov 5];14(3):289-95. Available from: https:// periodicos.ufff.br/index.php/aps/article/view/14607

3. Andrade MV, Maia AC, Cardoso CS, Alkmim MB, Ribeiro ALP. Custo-benefício do serviço de telecardiologia no Estado de Minas Gerais: projeto Minas Telecardio. Arq. Bras. Cardiol. [Internet]. 2011 [cited 2018 Jun 13];97(4):307-16. Available from: https://www.readcube.com/articles $/ 10.1590 \% 2 F s 0066-782 \times 2011005000080$

4. Andrade MV, Maia AC, Cardoso CS, Alkmim MB, Ribeiro ALP. Custo-Benefício do Serviço de Telecardiologia no Estado de Minas Gerais: Projeto Minas Telecardio. Arq. Bras. Cardiol. [Internet]. 2011 [cited 2018 Jun 13];97(4):307-16. doi: https://doi.org/10.1590/S0066$782 \times 2011005000080$

5. Universidade Federal de Minas Gerais. Hospital das Clínicas. Centro de Telessaúde. História [internet]. Belo Horizonte: UFMG, 2018 [cited 2018 Jun 13]. Available from: http://www.telessaude.hc.ufmg.br/quem-somos/ historial

6. Bortoluzzi ECB, Cardoso PH, Duarte RO, Madeira K. Alterações encontradas em eletrocardiogramas de repouso: prevalência e perfil epidemiológico. Arq. Catarin. Med. [Internet]. 2012 [cited 2018 Jun 13];41(3):14-9. Available from: http://www.acm.org.br/revista/pdf/artigos/938.pdf

7. Souza KT, Silva TMD. Relato de caso: a importância da educação permanente na realização de exames de ECG em Telessaúde [Resumo simples]. J. Bras. Tele. [Internet]. 2013 [cited 2018 Jun 13];2(Supl. 1):200. Available from: http://www.telessaude.uerj.br/jornal/volume/download_artigo/594

8. Brasil. Ministério da Saúde. Secretaria de Vigilância em Saúde. Departamento de Vigilância de Doenças e Agravos Não Transmissiveis e Promoção da Saúde. Saúde Brasil 2017 - uma análise da situação de saúde e os desafios para o alcance dos objetivos de desenvolvimento sustentável. Brasilia: MS, 2018. 426p. Available from: https://bvsms.saude.gov.br/bvs/ publicacoes/saude_brasil_2017_analise_situacao_saude_desafios_objetivos_desenvolvimento_sustetantavel.pdf

9. Scherr C, Ribeiro JP. Gênero, idade, nível social e fatores de risco cardiovascular: considerações sobre a realidade brasileira. Arq. Bras. Cardiol. [Internet]. 2009 [cited 2018 Jun 13];93(3):54-6. doi: https://doi. org/10.1590/50066-782X2009000900022

10. Bacquer $D$, Backer $G$, Kornitzer $M$. Prevalences of ECG findings in large population based samples of men and women. Heart [Internet]. 2000 [cited 2018 Jun 31];84(6):625-33. doi: http://doi.org/10.1136/heart.84.6.625

11. Santos RD (col.). III Diretrizes brasileiras sobre dislipidemias e diretriz de prevenção da aterosclerose do Departamento de Aterosclerose da Sociedade Brasileira de Cardiologia. Arq. Bras. Cardiol. [Internet]. 2001 [cited 2018 Jun 13];77(supl. 3):1-48. doi: https://doi.org/10.1590/50066782X2001001500001

12. Rique $A B R$, Soares $E A$, Meirelles $C M$. Nutrição e exercício na prevenção e controle das doenças cardiovasculares. Rev. Bras. Med. Esporte [Internet]. 2002 [cited 2018 Jun 13];8(6):244-54. Available from: https:// www.scielo.br/pdf/rbme/v8n6/v8n6a06.pdf

13. Martín JJD, Diéguez IM, Luis JA, Junquera MAD, Vázquez MV, Guerrero SM. Agrupamiento de factores de riesgo cardiovascular en hijos obesos de padres con hipertensión esencial. An. Pediatr. [Internet]. 2005 [cited 2018 Jun 13];63(3):238-43. doi: https://doi.org/10.1157/13078487

14. Haddad N, Bittar OJNV, Pereira AAM, Silva MB, Amato VL, Farsky $\mathrm{PS}$, et al. Conseqüências do prolongamento do tempo de espera em pacientes candidatos à cirurgia cardíaca. Arq. Bras. Cardiol. [Internet]. 2002 [cited 2018 Jun 13];78(5):452-8. doi: http://doi.org/10.1590/S0066$782 \times 2002000500003$

15. Cardoso E, Martins IS, Fornari L, Monachini MC, Mansur AP, Caramelli $B$. Alterações eletrocardiográficas e sua relação com os fatores de risco para doença isquêmica do coração em população da área metropolitana de São Paulo. Rev. Assoc. Med. Bras. [Internet]. 2002 [cited 2018 Jun 13];48(3):231-6. doi: https://doi.org/10.1590/S010442302002000300036 